\title{
The Incidence and Mortality of Liver Cancer and its Relationship with Development in Asia
}

\author{
Maryam Mohammadian ${ }^{1}$, Ali Soroush ${ }^{2}$, Abdollah Mohammadian-Hafshejani ${ }^{3}$, \\ Farhad Towhidi ${ }^{4}$, Fatemeh Hadadian ${ }^{5}$, Hamid Salehiniya ${ }^{6,7 *}$
}

\begin{abstract}
Background: Liver cancer (LC) is the sixth world most common cancer and the second leading cause of cancer death. Due to the importance and necessity of awareness about the incidence and mortality of diseases to perform prevention programs, this study focused on data for $\mathrm{LC}$ and its relationship with the human development index (HDI) and its components in Asia in 2012. Materials and Methods: This ecological study was based on GLOBOCAN data for Asian countries. We assessed correlations between standardized incidence rates (SIR) and standardized mortality rates (SMR) of LC with HDI and its components using of SPSS18. Results: A total of 582,420 incident cases and 557,097 deaths were recorded in Asian countries in 2012. The five with the highest SIR were Mongolia, Lao PDR, Vietnam, Republic of Korea and Thailand and those with the highest SMR were Mongolia, Lao PDR, Vietnam, Cambodia and Thailand. A negative relation was observed between HDI and LC for SIR of $0.049(\mathrm{P}=0.748)$ and for SMR of $0.07(\mathrm{P}=\mathbf{0 . 6 4 5})$, with life expectancy at birth a positive relation for SIR of $0.061(P=0.687)$ and a negative relation for $S M R$ of $0.079(P=0.603)$, with the average years of education a negative relation fo SIR of $0.476(\mathrm{p}=\mathbf{0 . 9 5 2})$ and for SMR of $0.032(\mathrm{P}=\mathbf{0 . 8 3 2})$, and with the country income level per person a negative relation for SMI of $0.11(p=0.465)$ and for SMR of $0.113(P=0.455)$. Conclusions: The incidence of $\mathrm{LC}$ is more in less developed and developing countries but statistically significant correlations were not found between standardized incidence and mortality rates of LC, and HDI and its dimensions.
\end{abstract}

Keywords: Human development index - liver cancer - incidence - mortality - Asia - epidemiology

Asian Pac J Cancer Prev, 17 (4), 2041-2047

\section{Introduction}

Cancer in 2013 was among the main leading causes of illness and death worldwide with an annual incidence of 14.9 million new cases and 8.2 million deaths caused. More than 60 percent occurs in Africa, Asia and Central and South America (Fitzmaurice et al., 2015). It is expected that the number of new cancer cases increases 70 percent in next two decades (Pakzad et al., 2015b).

Liver cancer is among the most common cancers in the world (Wei et al., 2014) so that this cancer is the fifth most common cancer in men (Hall and Wild, 2003) and the ninth most common cancer in women worldwide. This cancer is the second most common cause of cancer death worldwide in 2012 (Pakzad et al., 2015b). Liver cancer mortality is rapidly increasing since 1970 . Due to this point that more than 85 percent of liver cancer new cases occur in developing countries which do not have proper diagnostic and treatment facilities (Ferlay et al., 2010), finally, the case fatality rate will rise so that the 5-year mortality rate of the disease is about $95 \%$ in the world (Hall and Wild, 2003). The Age Standardized Incidence Rates (ASIRs) per 100 thousand people for liver cancer is 15 to 7 in developing countries than in developed ones in 2013 and the Age Standardized Death Rates (ASDRs) is 16 to 7 for it (Fitzmaurice et al., 2015).

The west region of Pacific Ocean has the highest incidence and mortality of liver cancer in the world. So that the incidence and mortality of liver cancer is about 38 times higher than the East Mediterranean sea which is the region with the lowest incidence and mortality rates. The successful experiences of world countries in a massive reduction in cancer incidence and mortality, even among low-income countries, shows that the Asia and Pacific Region significantly have the potential to reduce the burden of cancer by appropriate policy and planning

${ }^{1}$ Health Promotion Research Center, Department of Epidemiology and Biostatistics, School of Public Health, Zahedan University of Medical Sciences, Zahedan, ${ }^{2}$ Lifestyle Modification Research Center, Imam Reza Hospital, Kermanshah University of Medical Sciences, Kermanshah, ${ }^{4}$ Imam Reza Hospital, ${ }^{5}$ Department of Medical Surgical Nursing, School of Nursing and Midwifery, Kermanshah University of Medical Sciences, Kermanshah, ${ }^{3}$ Department of Social Medicine, School of Medicine, Rafsanjan University of Medical Sciences, Rafsanjan, ${ }^{6}$ Zabol University of Medical Sciences, Zabol, ${ }^{7}$ Department of Epidemiology \& Biostatistics, School of Public Health, Tehran University of Medical Sciences, Tehran, Iran*For correspondence: alesaleh70@yahoo.com 
(Ferlay et al., 2010).

The United Nations Development Program (UNDP) has used the Human Development Index (HDI) for the first time in 1990 in order to prevent income concentration and the move towards a more comprehensive measure of human development (Dasgupta and Weale, 1992). The HDI is an index composed of three basic dimensions: human development, including a long and healthy life (Based on life expectancy at birth), access to knowledge (based on a combination of the adult literacy rate and enrollment in primary and high-level education) and the suitable life standard (based on the Gross Domestic Product due to the equality of purchasing power).

Overall, HDI is the arithmetic average of the three indicators calculated for each country individually (Sagar and Najam, 1998; Mahdavifar et al., 2016). It seems that the Human Development Index and its components have correlation with the incidence and mortality of liver cancer, so that the results of various studies are the indicators of the relationship between the incidence and mortality of some of the cancers with the development index (Ghoncheh et al., 2015a; Ghoncheh et al., 2015b; Pakzad et al., 2015c; Pakzad et al., 2015a; Razi et al, 2016). Do to this point that the awareness about the incidence and mortality of this cancer is essential in planning, this study is done with the aim to investigate the incidence and mortality of liver cancer and its relationship with Human Development Index in Asian 2012.

\section{Materials and Methods}

This study was an ecologic study in Asia for assessment the correlation between age-specific incidence and mortality rate (ASR) with Human Development Index (HDI) and its details that include: Life expectancy at birth, Mean years of schooling and Gross national income (GNI) per capita. Data about the age-specific incidence and mortality rate (ASR) for every Asian counter for year 2012 get from global cancer project that available in (http://globocan.iarc.fr/Default.aspx) (Ferlay J et al., 2016) and Human Development Index (HDI) from Human Development Report 2013 (Malik, 2013) that include information about HDI and its details for every country in the word for year 2012.

Method of estimate the age-specific incidence and mortality rates in global cancer project by international agency for research on cancer

\section{Age-specific incidence rate estimate}

The methods of estimation are country specific and the quality of the estimation depends upon the quality and on the amount of the information available for each country. In theory, there are as many methods as countries, and because of the variety and the complexity of these methods, an overall quality score for the incidence and mortality estimates combined is almost impossible to establish. However an alphanumeric scoring system which independently describes the availability of incidence and mortality data has been established at the country level. The combined score is presented together with the estimates for each country with an aim of providing a broad indication of the robustness of the estimation.

The methods to estimate the sex- and age-specific incidence rates of cancer for a specific country fall into one of the following broad categories, in priority order:

1- Rates projected to 2012 (38 countries)-2- Most recent rates applied to 2012 population (20 countries)3-Estimated from national mortality by modelling, using incidence mortality ratios derived from recorded data in country-specific cancer registries (13 countries)-4Estimated from national mortality estimates by modelling, using incidence mortality ratios derived from recorded data in local cancer registries in neighboring countries (9 European countries)-5-Estimated from national mortality estimates using modelled survival (32 countries)-6Estimated as the weighted average of the local rates (16 countries)-7- One cancer registry covering part of a country is used as representative of the country profile (11 countries)-8-Age/sex specific rates for "all cancers" were partitioned using data on relative frequency of different cancers (by age and sex) (12 countries)-9- The rates are those of neighboring countries or registries in the same area (33 countries) (Ferlay et al., 2015; Ferlay J et al., 2016).

\section{Age-specific mortality rate estimate}

Depending of the degree of detail and accuracy of the national mortality data, six methods have been utilized in the following order of priority:

1-Rates projected to 2012 (69 countries)-2- Most recent rates applied to 2012 population (26 countries)-3Estimated as the weighted average of regional rates (1 country)-4- Estimated from national incidence estimates by modelling, using country-specific survival ( 2 countries)-5-Estimated from national incidence estimates using modelled survival (83 countries)-6-The rates are those of neighboring countries or registries in the same area (3 countries) (Ferlay et al., 2015; Ferlay J et al., 2016).

\section{Human Development Index (HDI)}

Human Development Index (HDI), a composite measure of indicators along three dimensions: life expectancy, educational attainment and command over the resources needed for a decent living. All groups and regions have seen notable improvement in all HDI components, with faster progress in low and medium HDI countries. On this basis, the world is becoming less unequal. Nevertheless, national averages hide large variations in human experience. Wide disparities remain within countries of both the North and the South, and income inequality within and between many countries has been rising. (Malik, 2013)

Statistical analysis: In this study, we use of correlation bivariate method for assessment the correlation between age-specific incidence and mortality rate (ASR) with Human Development Index (HDI) and its details that include: Life expectancy at birth, Mean years of schooling and Gross national income (GNI) per capita. Statistical significance was assumed if $\mathrm{P}<0.05$. All reported $\mathrm{P}$-values are two-sided. Statistical analyses were performed using SPSS (Version 15.0, SPSS Inc). 


\section{Results}

Overall, in 2012, 582420 cases of liver cancer in Asian countries have been registered, of which 422317 cases $(51 / 72 \%)$ were in men and 160103 cases $(48 / 27 \%)$ were in women. The sex ratio of disease is 2.63 . In Asia 5 countries with the highest numbers of liver cancer new cases are as follows, respectively: 1. China with 394,770 cases, 2. Japan with 36,168 cases, 3.India with 27,416 cases, 4 . Vietnam with 21,997cases and 5. Thailand with 20,455 cases. These five countries have allocated a total of 500,806 cases $(98 / 85 \%)$.

Asia

In Asian countries, 5 countries having the highest standardized incidence rate of liver cancer are as follows, respectively: 1. Mongolia with the standardized incidence rate of 78.1 per hundred thousand people, 2.Lao PDR with the rate of 52.6 per hundred thousand people, 3 . Vietnam with the rate of 24.6 per hundred thousand people, 4 . Republic of Korea with the rate of 22.8per hundred thousand people and 5.Thailand with the rate of 22.3 per hundred thousand people. Similarly, 5 countries having the lowest standardized incidence rate of liver cancer are as follows: 1 . Nepal with the rate of 0.9 per hundred thousand people, 2. Israel with the rate of 2.3 per hundred thousand people, 3 . Islamic Republic of Iran with the rate of 2.5 per hundred thousand people, 4 . Lebanon with the rate of 2.6 per hundred Thousand people and 5. India with the rate of 2.7 per hundred thousand people.

The number and amount of crude and standardized incidence rate of this cancer is presented in table one due to the sex in Asian countries. Countries are arranged based on standardized rate from high to low in Table one. So in each sex, we can see countries with the highest and lowest standardized rate. (Figure and table 1)

In addition, during 2012 the number of 557097 deaths due to liver cancer occurred in Asia of which 400835 cases $(71.95 \%)$ were in men and 156262 cases $(28.4 \%)$ occurred in women.

The sex ratio of disease is 2.56 . The highest death number occurred in: china with the number of 383203 cases, Japan with the number of 32518 cases, India with the number of 26763 cases, Vietnam with the number of

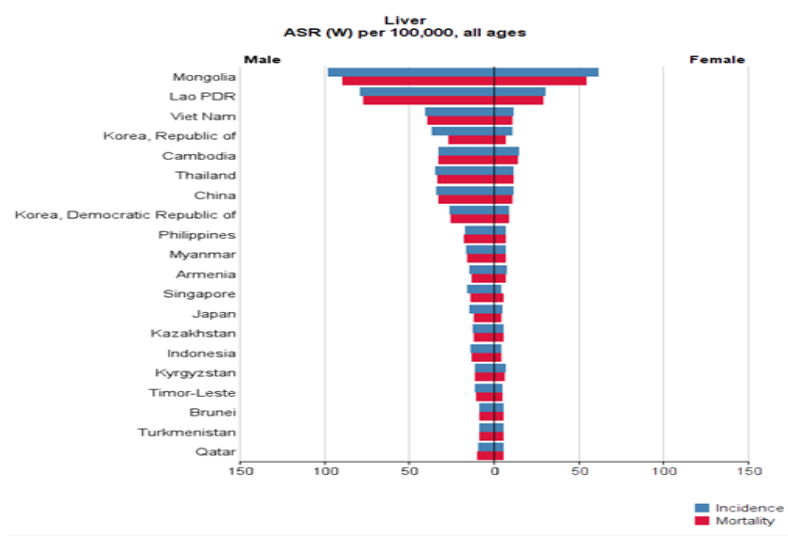

Figure 1.Standardized Incidence and Mortality Rates for Liver Cancer in Asia in 2012
20920 cases and Thailand with the number of 19442 cases. A total of 482846 cases $(86.67 \%)$ of the deaths occurred in just these five countries.

In Asian countries, 5 countries having the highest standardized mortality rate of liver cancer are as follows, respectively: 1. Mongolia with the standardized rate of 70.3 per Hundred thousand people, 2.Lao PDR with the rate of 50.9 per hundred thousand people, 3. Vietnam with the rate of 23.7 per hundred thousand people, 4 . Cambodia with the rate of 21.5 Per hundred thousand people and 5. Thailand with the rate of 21.5 per hundred thousand people. Similarly, 5 countries having the lowest standardized mortality rate of liver cancer are as follows: 1. Nepal with the rate of 0.9 per hundred thousand people, 2.Islamic Republic of Iran with the rate of 2.3 per hundred thousand people, 3. Lebanon with the rate of 2.4 per hundred thousand people, 4 .Israel with the rate of 2.5 per hundred thousand people and 5. India with the rate of 2.6 per hundred thousand people (Figure 1).

In Table 2, values related to the Human Development Index and its components for each of the Asian countries are shown and arranged based on the HDI (human development index).The Asian countries in terms of Human Development Index are classified as follows so that three countries are classified in very top category, four countries in top category, thirty-five countries in medium category, three countries in low category and one country in unknown category.

Standardized incidence rate and the human development index

It has been seen a negative correlation of 0.049

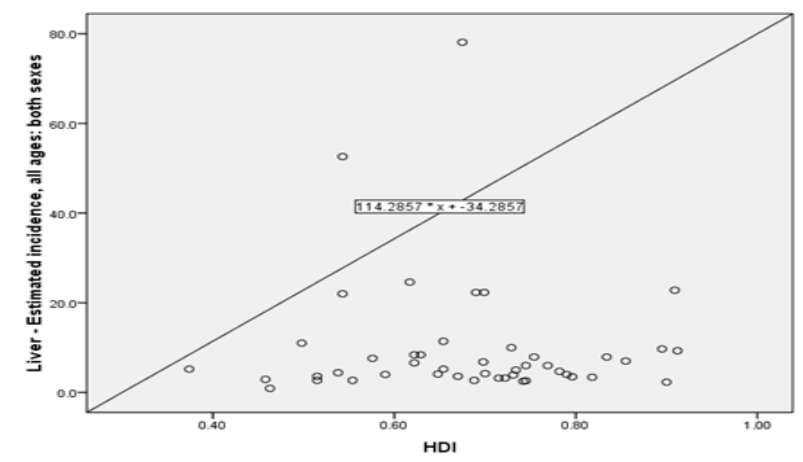

Figure 2. Correlation between the Human Development Index and Standardized Incidence Rate of Liver Cancer in Asia in 2012

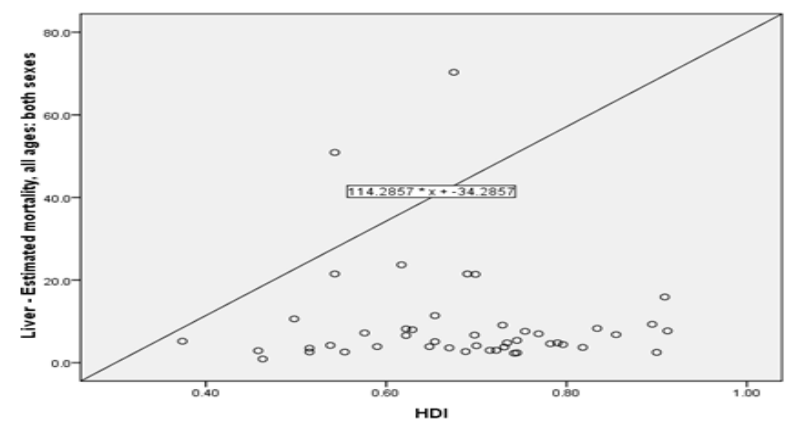

Figure 3. Correlation between the Human Development Index and Standardized Mortality Rate of Liver Cancer in Asia in 2012 
Table 1. Number and Amount of Crude and Standardized incidence rate of liver Cancer in Asian Countries in 2012(Sorted by Age-Standardized Rate from the Highest to the Lowest Values

\begin{tabular}{|c|c|c|c|c|c|c|c|c|c|c|c|}
\hline \multicolumn{4}{|c|}{$\begin{array}{l}\text { Liver- Estimated incidence, all ages: both } \\
\text { sexes }\end{array}$} & \multicolumn{4}{|c|}{ Liver- Estimated incidence, all ages: male } & \multicolumn{4}{|c|}{$\begin{array}{l}\text { Liver- Estimated incidence, all ages: } \\
\text { female }\end{array}$} \\
\hline 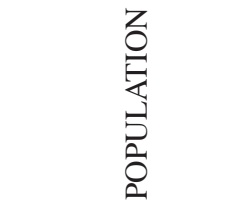 & 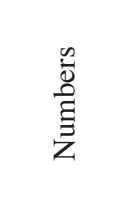 & 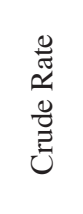 & $\begin{array}{l}\sum \\
\frac{1}{2} \\
\varepsilon\end{array}$ & 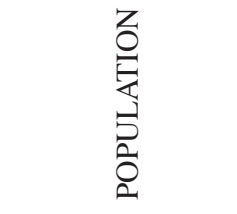 & 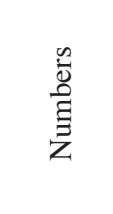 & 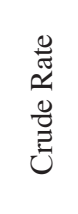 & $\underset{\approx}{\approx}$ & 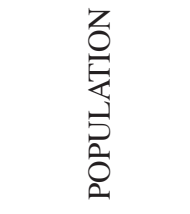 & $\begin{array}{l}\text { 氙 } \\
\text { है } \\
\text { Z }\end{array}$ & 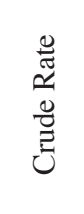 & \begin{tabular}{l}
$\sum$ \\
\multirow{2}{*}{} \\
\multirow{2}{*}{}
\end{tabular} \\
\hline Mongolia & 1518 & 53.4 & 78.1 & Mongolia & 888 & 63.3 & 97.8 & Mongolia & 630 & 43.7 & 61.1 \\
\hline Lao PDR & 2116 & 33.2 & 52.6 & Lao PDR & 1486 & 46.7 & 78.7 & Lao PDR & 630 & 19.7 & 29.7 \\
\hline Viet Nam & 21997 & 24.5 & 24.6 & Viet Nam & 16815 & 37.9 & 40.2 & Cambodia & 820 & 11.1 & 14.2 \\
\hline South Korea & 16900 & 34.8 & 22.8 & South Korea & 12559 & 51.9 & 36.7 & Thailand & 5716 & 16.1 & 11.3 \\
\hline Thailand & 20455 & 29.3 & 22.3 & Thailand & 14739 & 42.9 & 34.8 & Viet Nam & 5182 & 11.4 & 10.9 \\
\hline China & 394770 & 29 & 22.3 & China & 293318 & 41.5 & 33.7 & China & 101452 & 15.5 & 10.9 \\
\hline Cambodia & 2264 & 15.6 & 22 & Cambodia & 1444 & 20.4 & 32.7 & South Korea & 4341 & 17.8 & 10.5 \\
\hline North Korea & 4969 & 20.2 & 16.2 & North Korea & 3391 & 28.1 & 25.8 & Norht Korea & 1578 & 12.6 & 8.7 \\
\hline Philippines & 7734 & 8 & 11.4 & Philippines & 5441 & 11.2 & 17.1 & Armenia & 204 & 12.3 & 6.9 \\
\hline Myanmar & 4912 & 10.1 & 11 & Myanmar & 3421 & 14.2 & 16.1 & Philippines & 2293 & 4.8 & 6.5 \\
\hline Armenia & 464 & 14.9 & 10 & Singapore & 588 & 22.2 & 15.8 & Myanmar & 1491 & 6 & 6.4 \\
\hline Singapore & 763 & 14.5 & 9.7 & Japan & 23651 & 38.4 & 14.6 & Kyrgyzstan & 138 & 5 & 6.3 \\
\hline Japan & 36168 & 28.6 & 9.3 & Armenia & 260 & 18 & 14.4 & Brunei & 6 & 2.9 & 5.4 \\
\hline Kyrgyzstan & 324 & 5.9 & 8.4 & Indonesia & 13365 & 11 & 13.4 & Kazakhstan & 547 & 6.4 & 5.3 \\
\hline Indonesia & 18121 & 7.4 & 8.4 & Kazakhstan & 790 & 10 & 12.2 & Turkmenistan & 110 & 4.2 & 5.2 \\
\hline Kazakhstan & 1337 & 8.2 & 7.9 & Timor-Leste & 29 & 4.8 & 11.1 & Tajikistan & 117 & 3.2 & 5.1 \\
\hline Qatar & 49 & 2.5 & 7.9 & Kyrgyzstan & 186 & 6.9 & 11 & Qatar & 5 & 1.1 & 5 \\
\hline Timor-Leste & 44 & 3.7 & 7.6 & Qatar & 44 & 3 & 8.9 & Japan & 12517 & 19.3 & 4.7 \\
\hline Brunei & 20 & 4.8 & 7 & Turkmenistan & 153 & 6 & 8.8 & Timor-Leste & 15 & 2.6 & 4.6 \\
\hline Turkmenistan & 263 & 5.1 & 6.8 & Malaysia & 1111 & 7.5 & 8.7 & Uzbekistan & 481 & 3.4 & 4.2 \\
\hline Tajikistan & 273 & 3.9 & 6.6 & Brunei & 14 & 6.7 & 8.7 & Singapore & 175 & 6.7 & 4.1 \\
\hline Malaysia & 1527 & 5.2 & 6 & Tajikistan & 156 & 4.5 & 8.5 & Indonesia & 4756 & 3.9 & 4 \\
\hline Georgia & 439 & 10.2 & 6 & Georgia & 250 & 12.3 & 8.5 & Azerbaijan & 212 & 4.5 & 3.9 \\
\hline Afghanistan & 760 & 2.3 & 5.2 & Afghanistan & 477 & 2.8 & 6.6 & Georgia & 189 & 8.3 & 3.9 \\
\hline Uzbekistan & 1078 & 3.8 & 5.2 & Azerbaijan & 254 & 5.4 & 6.4 & Afghanistan & 283 & 1.8 & 3.8 \\
\hline Azerbaijan & 466 & 4.9 & 5 & Saudi Arabia & 516 & 3.3 & 6.4 & Iraq & 360 & 2.1 & 3.7 \\
\hline Saudi Arabia & 722 & 2.5 & 4.7 & Uzbekistan & 597 & 4.3 & 6.3 & Bahrain & 9 & 1.8 & 3.4 \\
\hline Bhutan & 25 & 3.3 & 4.4 & Bhutan & 18 & 4.5 & 6.1 & Syria & 244 & 2.3 & 3.3 \\
\hline Jordan & 159 & 2.5 & 4.2 & Jordan & 100 & 3 & 5.4 & Malaysia & 416 & 2.9 & 3.3 \\
\hline Syria & 565 & 2.7 & 4.1 & Kuwait & 43 & 2.5 & 5.3 & Jordan & 59 & 1.9 & 3 \\
\hline Kuwait & 52 & 1.8 & 4 & Maldives & 6 & 3.7 & 5.3 & UAE & 17 & 0.7 & 3 \\
\hline Iraq & 678 & 2 & 4 & State of Palestine & 50 & 2.3 & 5.1 & Saudi Arabia & 206 & 1.6 & 2.8 \\
\hline Oman & 59 & 2 & 3.9 & Syria & 321 & 3 & 4.9 & Oman & 17 & 1.4 & 2.8 \\
\hline State of Palestine & 73 & 1.7 & 3.6 & Pakistan & 2824 & 3.1 & 4.7 & Pakistan & 1500 & 1.7 & 2.5 \\
\hline Pakistan & 4324 & 2.4 & 3.6 & Turkey & 1537 & 4.1 & 4.7 & Bhutan & 7 & 2 & 2.4 \\
\hline Bahrain & 26 & 1.9 & 3.5 & Oman & 42 & 2.4 & 4.6 & $\begin{array}{l}\text { State of } \\
\text { Palestine }\end{array}$ & 23 & 1.1 & 2.2 \\
\hline UAE & 68 & 0.8 & 3.4 & Sri Lanka & 527 & 5 & 4.4 & Iran & 678 & 1.8 & 2.1 \\
\hline Sri Lanka & 806 & 3.8 & 3.2 & Iraq & 318 & 1.9 & 4.4 & Bangladesh & 1185 & 1.6 & 2.1 \\
\hline Turkey & 2227 & 3 & 3.2 & Yemen & 222 & 1.7 & 4.1 & Sri Lanka & 279 & 2.6 & 2.1 \\
\hline Yemen & 349 & 1.4 & 2.9 & Bahrain & 17 & 2 & 3.6 & Yemen & 127 & 1 & 2 \\
\hline Maldives & 6 & 1.9 & 2.7 & UAE & 51 & 0.9 & 3.5 & Lebanon & 47 & 2.1 & 1.9 \\
\hline Bangladesh & 3022 & 2 & 2.7 & India & 17236 & 2.7 & 3.5 & India & 10180 & 1.7 & 1.9 \\
\hline India & 27416 & 2.2 & 2.7 & Israel & 158 & 4.2 & 3.4 & Kuwait & 9 & 0.8 & 1.9 \\
\hline Lebanon & 116 & 2.7 & 2.6 & Bangladesh & 1837 & 2.4 & 3.3 & Turkey & 690 & 1.8 & 1.8 \\
\hline Iran & 1567 & 2.1 & 2.5 & Lebanon & 69 & 3.3 & 3.3 & Israel & 85 & 2.2 & 1.3 \\
\hline Israel & 243 & 3.2 & 2.3 & Iran & 889 & 2.3 & 2.8 & Nepal & 77 & 0.5 & 0.7 \\
\hline Nepal & 186 & 0.6 & 0.9 & Nepal & 109 & 0.7 & 1.2 & Maldives & 0 & 0 & 0 \\
\hline
\end{tabular}

between the standardized incidence rate of liver cancer and the Human Development Index that this correlation is not statistically significant $(\mathrm{p}=0.748)$. Also it was seen an insignificant correlation between the human development index components with standardized rate. So that it was seen a positive correlation of 0.061 between the standardized incidence rate and life expectancy at birth ( $\mathrm{p}=0.687)$, negative correlation of 0.009 with mean years of schooling $(\mathrm{p}=0.952)$ and negative correlation of 0.11 with income level of the population per person ( $\mathrm{p}=0.465)$.

In men also, It has been seen a negative correlation of 0.047 between the standardized incidence rate of liver 
Table 2. The Human Development Index in Asian Countries in 2012

\begin{tabular}{|c|c|c|c|c|c|}
\hline HDI situation & POPULATION & $\begin{array}{l}\text { Human Development } \\
\text { Index(HDI) }\end{array}$ & $\begin{array}{l}\text { Life expectancy } \\
\text { at birth }\end{array}$ & $\begin{array}{l}\text { Mean Year of } \\
\text { schooling }\end{array}$ & $\begin{array}{l}\text { Gross national income } \\
\text { (GNI) per capita }\end{array}$ \\
\hline \multirow[t]{3}{*}{ Very high } & Japan & 0.912 & 83.6 & 11.6 & 32545 \\
\hline & Korea, Republic of & 0.909 & 80.7 & 11.6 & 28231 \\
\hline & Israel & 0.9 & 81.9 & 11.9 & 26224 \\
\hline \multirow[t]{4}{*}{ High } & Singapore & 0.895 & 81.2 & 10.1 & 52613 \\
\hline & Brunei & 0.855 & 78.1 & 8.6 & 45690 \\
\hline & Qatar & 0.834 & 78.5 & 7.3 & 87478 \\
\hline & United Arab Emirates & 0.818 & 76.7 & 8.9 & 42716 \\
\hline \multirow[t]{35}{*}{ Medium } & Bahrain & 0.796 & 75.2 & 9.4 & 19154 \\
\hline & Kuwait & 0.79 & 74.7 & 6.1 & 52793 \\
\hline & Saudi Arabia & 0.782 & 74.1 & 7.8 & 22616 \\
\hline & Malaysia & 0.769 & 74.5 & 9.5 & 13676 \\
\hline & Kazakhstan & 0.754 & 67.4 & 10.4 & 10451 \\
\hline & Georgia & 0.745 & 73.9 & 12.1 & 5005 \\
\hline & Lebanon & 0.745 & 72.8 & 7.9 & 12364 \\
\hline & Iran, Islamic Republic of & 0.742 & 73.2 & 7.8 & 10695 \\
\hline & Azerbaijan & 0.734 & 70.9 & 11.2 & 8153 \\
\hline & Oman & 0.731 & 73.2 & 5.5 & 24092 \\
\hline & Armenia & 0.729 & 74.4 & 10.8 & 5540 \\
\hline & Turkey & 0.722 & 74.2 & 6.5 & 13710 \\
\hline & Sri Lanka & 0.715 & 75.1 & 9.3 & 5170 \\
\hline & Jordan & 0.7 & 73.5 & 8.6 & 5272 \\
\hline & China & 0.699 & 73.7 & 7.5 & 7945 \\
\hline & Turkmenistan & 0.698 & 65.2 & 9.9 & 7782 \\
\hline & Thailand & 0.69 & 74.3 & 6.6 & 7722 \\
\hline & Maldives & 0.688 & 77.1 & 5.8 & 7478 \\
\hline & Mongolia & 0.675 & 68.8 & 8.3 & 4245 \\
\hline & State of Palestine & 0.67 & 73 & 8 & 3359 \\
\hline & Philippines & 0.654 & 69 & 8.9 & 3752 \\
\hline & Uzbekistan & 0.654 & 68.6 & 10 & 3201 \\
\hline & Syrian Arab Republic & 0.648 & 76 & 5.7 & 4674 \\
\hline & Indonesia & 0.629 & 69.8 & 5.8 & 4154 \\
\hline & Kyrgyzstan & 0.622 & 68 & 9.3 & 2009 \\
\hline & Tajikistan & 0.622 & 67.8 & 9.8 & 2119 \\
\hline & Viet Nam & 0.617 & 75.4 & 5.5 & 2970 \\
\hline & Iraq & 0.59 & 69.6 & 5.6 & 3557 \\
\hline & Timor-Leste & 0.576 & 62.9 & 4.4 & 5446 \\
\hline & India & 0.554 & 65.8 & 4.4 & 3285 \\
\hline & Cambodia & 0.543 & 63.6 & 5.8 & 2095 \\
\hline & Lao PDR & 0.543 & 67.8 & 4.6 & 2435 \\
\hline & Bhutan & 0.538 & 67.6 & 2.3 & 5246 \\
\hline & Bangladesh & 0.515 & 69.2 & 4.8 & 1785 \\
\hline & Pakistan & 0.515 & 65.7 & 4.9 & 2566 \\
\hline \multirow[t]{4}{*}{ Low } & Myanmar & 0.498 & 65.7 & 3.9 & 1817 \\
\hline & Nepal & 0.463 & 69.1 & 3.2 & 1137 \\
\hline & Yemen & 0.458 & 65.9 & 5.3 & 928 \\
\hline & Afghanistan & 0.374 & 49.1 & 3.1 & 1000 \\
\hline Unknown & North Korea & - & - & - & - \\
\hline
\end{tabular}

cancer and the Human Development Index that this correlation is not statistically significant $(\mathrm{p}=0.755)$. Also it was seen a negative correlation between the Human Development index components with standardized rate. So that it was seen a negative correlation of 0.041 between the standardized incidence rate and life expectancy at birth $(\mathrm{p}=0.786)$ in men, negative correlation of 0.016 with mean years of schooling $(\mathrm{p}=0.916)$ and negative correlation of 0.119 with income level of the population per person $(\mathrm{p}=0.429)$.

In women also, it has been seen a negative correlation of 0.061 between the standardized incidence rate of liver cancer and the Human Development Index but this correlation is not statistically significant $(\mathrm{p}=0.687)$. Also it was seen a correlation between the human development index components with standardized rate. So that it was seen a positive correlation of 0.109 between the standardized incidence rate and life expectancy at birth ( $\mathrm{p}=0.470)$, positive correlation of 0.012 with mean years of schooling $(\mathrm{p}=0.935)$ and also a negative correlation of 0.120 with the income level of the population per person $(\mathrm{p}=0.426)$.

Standardized mortality rate and the human development index

Also it has been seen a negative correlation of 0.07 between the standardized mortality rate of liver cancer and the Human Development Index but this correlation 
is not statistically significant $(\mathrm{p}=0.645)$. Also it was seen a correlation between the HDI components with standardized rate. So that it was seen a negative correlation of 0.079 between the standardized mortality rate and life expectancy at birth $(\mathrm{p}=0.603)$, negative correlation of 0.032 with mean years of schooling $(p=0.832)$ and negative correlation of 0.113 with income level of the population per person $(\mathrm{p}=0.455)$.

In men also, It has been seen a negative correlation of 0.076 between the standardized mortality rate of liver cancer and the HDI that this correlation is not statistically significant $(\mathrm{p}=0.615)$. Also it was seen a correlation between the Human development index components with standardized rate. So that it was seen a negative correlation of 0.067 between the standardized mortality rate and life expectancy at birth $(\mathrm{p}=0.660)$, negative correlation of 0.041 with mean years of schooling $(p=0.789)$ and negative correlation of 0.134 with income level of the population per person $(\mathrm{p}=0.374)$.

In women also, it has been seen a negative correlation of 0.069 between the standardized incidence rate of liver cancer and the HDI that this correlation is not statistically significant $(\mathrm{p}=0.649)$. Also it was seen a correlation between the HDI components with standardized rate. So that it was seen a negative correlation of 0.116 between the standardized mortality rate and life expectancy at birth ( $\mathrm{p}=0.441)$, negative correlation of 0.007 with mean years of schooling $(\mathrm{p}=0.965)$ and negative correlation of 0.104 with income level of the population per person $(\mathrm{p}=0.490)$.

\section{Discussion}

In recent years, the greatest burden of cancer is seen in developing countries. So that now about $57 \%$ of cancer cases and 65 percent of deaths due to that occur in these countries worldwide (Bray and Moller, 2006). So cancer is becoming a serious health threat in developing regions especially in many Asian countries and as one of the main leading causes of death in some Asian countries such as Japan and South Korea. It is predicted that if management strategies, in these areas will continue to be stable, the number of new cancer cases in Asia will rise to 7.1 million by 2020 (Mackay J, (2006).).

Based on the findings of this study, the incidence of liver cancer in Asia is not in relation with The Human Development Index which checks a countries' mean success in three Human dimensions: Long life, access to knowledge and a desirable life standard. Among Asian countries, Mongolia, Lao PDR, Vietnam, the Republic of Korea and Thailand had the highest standardized incidence of cancer. The Human Development Index in 4 countries is in medium level but in Republic of Korea, its level is too high. In contrast, in countries such as Nepal, Israel, Islamic Republic of Iran, Lebanon and India with very high, medium and low human development index low incidence was seen. The results of another study showed that although hepatocellular carcinoma (HCC) due to chronic hepatitis B infection is more common in Asia and Africa that have lower HDI level, HCC rates due to vaccination programs in some areas have declined in recent years (Chang et al., 1997) .
It seems that the rise of $\mathrm{HCC}$ in Western countries that have a higher HDI, has been due to hepatitis $\mathrm{C}$ infection and alcohol consumption (Liver, 2012). Distribution of cancers based on HDI is different in the world. So that countries with high very high and high HDI have four types of cancers including lung cancer, breast, colorectal and prostate cancer have allocated almost half of the total cancer incidence load to themselves. In countries with medium and low HDI, in addition to lung, colorectal and breast cancer, cancers of the stomach, liver and cervical cancer have allocated a significant amount of cancer to themselves. In countries with low HDI, though the absolute burden of cancer is low, but a variety of cancers such as cancer of the cervix, liver, Kaposi's sarcoma and non-Hodgkin that the etiology of all of them is related to infectious agents, have the highest incidence and mortality rates in these areas (Bray et al., 2012; Franceschi and Wild, 2013).

The results of this study showed that the standardized mortality rate of liver cancer in Asia varies from 0.9 per hundred thousand people in Nepal to 70.3 per hundred thousand people in Mongolia. The most standardized mortality rates occurred in Mongolia, Lao PDR, Vietnam, Cambodia and Thailand with the average level of Human Development Index. And it is noteworthy that, countries with high incidence does not experience higher mortality necessarily.

The standardized mortality rate showed insignificant negative correlation with HDI and its markers like life expectancy at birth, mean education and level of life. Similarly, 5 countries having the lowest standardized mortality rate of liver cancer are: Nepal, Iran, Lebanon, Israel and India that are in low, medium and very high level in terms of HDI. While the results of another study showed that the ratio of mortality to incidence of digestive system cancer including liver has an inverse relation with HDI in national and regional levels (Hu et al., 2013).

In this study a positive correlation was seen between the standardized incidence rate and life expectancy at birth as one of the HDI dimensions. But this relation was not statistically significant. Also a negative insignificant correlation was seen between standardized mortality rates with life expectancy at birth. Studies results have shown that age in many malignant cancers has been mentioned as a prognostic item but it may have controversial role in the prognosis of HCC. Young patients with liver cancer have a poorer prognosis, because they show poor pathological grade and advanced stage of the tumor. However, some studies have argued while young patients with liver cancer have poor clinical manifestations, their long-term survival are better than older patients. These different results may be due to performing study in a single center and limitation of sample size (Ni et al., 1991; Tobe et al., 1994; Yamanaka et al., 1994; Lee and Ko, 1998; Zhang and Sun, 2015).

Another indicator of the Human Development Index is access to knowledge that in this study has an insignificant negative correlation with the standardized incidence rate of liver cancer. While other research in this area showed that one of the important primary prevention actions for HCC, is improving public health in countries which requires new training strategies and increasing awareness 
and knowledge of individuals which is expected to lead to improvements in early detection and prevention of new cases of HBV and HCC (He et al., 2013).

Therefore, the implementation of targeted cultural and educational programs with an emphasis on prevention and screening for HBV was considered as an important tool for the prevention of liver cancer (Chao et al., 2009).

Another dimension of the HDI is suitable income level which is determined by Gross domestic Product. In our study, income level had an insignificant negative correlation with standardized incidence and mortality rate. While other studies have shown that different Patterns exist in different countries with various geographical, cultural, social and economic status (Sepanlou et al., 2015). So that each year more cases of liver cancer is recognized in less developed countries, compared with more developed ones.

In conclusion, the incidence of LC is more in less developed and developing countries. Statistically significant correlation was not found between standardized incidence and mortality rates of LC, and HDI and its dimensions.

\section{References}

Bray F, Jemal A, Grey N, et al (2012). Global cancer transitions according to the Human Development Index (2008-2030): a population-based study. Lancet Oncol, 13, 790-801.

Bray F, Moller B (2006). Predicting the future burden of cancer. Nature Reviews Cancer, 6, 63-74.

Chang M-H, Chen C-J, Lai M-S, et al (1997). Universal hepatitis $B$ vaccination in Taiwan and the incidence of hepatocellular carcinoma in children. New Engl J Med, 336, 1855-9.

Chao SD, Chang ET, Le PV, et al (2009). The Jade Ribbon Campaign: a model program for community outreach and education to prevent liver cancer in Asian Americans. $J$ Immigrant Minority Health, 11, 281-90.

Dasgupta P, Weale M (1992). On measuring the quality of life. World Development, 20, 119-31.

Ferlay J, Soerjomataram I, Ervik M, et al (2016). GLOBOCAN 2012 v1.0, Cancer Incidence and Mortality Worldwide: IARC CancerBase No. 11 [Internet]. Lyon, France: International Agency for Research on Cancer; 2013.

Ferlay J, Shin HR, Bray F, et al (2010). Estimates of worldwide burden of cancer in 2008: GLOBOCAN 2008. International J Cancer, 127, 2893-917.

Ferlay J, Soerjomataram I, Dikshit R, et al (2015). Cancer incidence and mortality worldwide: sources, methods and major patterns in GLOBOCAN 2012. International $J$ Cancer, 136, 359-86.

Fitzmaurice C, Dicker D, Pain A, et al (2015). The global burden of cancer 2013. JAMA Oncol, 1, 505-27.

Franceschi S, Wild CP (2013). Meeting the global demands of epidemiologic transition-the indispensable role of cancer prevention. Molecular Oncol, 7, 1-13.

Ghoncheh M, Mirzaei M, Salehiniya H (2015a). Incidence and mortality of breast cancer and their relationship with the human development index (HDI) in the World in 2012. Asian Pac J Cancer Prev, 16, 8439-43.

Ghoncheh M, Mohammadian-Hafshejani A, Salehiniya H (2015b). Incidence and mortality of breast cancer and their relationship to development in Asia. Asian Pac J Cancer Prev, 16, 6081-7.

Hall AJ, Wild CP (2003). Liver cancer in low and middle income countries: prevention should target vaccination, contaminated needles, and aflatoxins. BMJ: British Medical $J, 326,994$.

He W-J, Xu M-Y, Xu R-R, et al (2013). Inpatients' knowledge about primary liver cancer and hepatitis. Asian Pacific $J$ Cancer Prev, 14, 4913-8.

Hu Q-D, Zhang Q, Chen W, et al (2013). Human development index is associated with mortality-to-incidence ratios of gastrointestinal cancers. World J Gastroenterol, 19, 5261.

Lee C-L, Ko Y-C (1998). Survival and distribution pattern of childhood liver cancer in Taiwan. European J Cancer, 34, 2064-7.

Liver EAFTSOT (2012). EASL-EORTC clinical practice guidelines: management of hepatocellular carcinoma. $J$ Hepatol, 56, 908-43.

Mackay J JA, Lee N, Parkin D, Eds. ( (2006).). The Cancer Atlas. American Cancer Society, Atlanta.

Mahdavifar N, Ghoncheh M, Pakzad R, et al (2016). Epidemiology, incidence and mortality of bladder cancer and their relationship with the development index in the World. Asian Pac J Cancer Prev, 17, 381-6.

Malik K (2013). Human development report 2013. The rise of the south: Human progress in a diverse world. The Rise of the South: Human Progress in a Diverse World (March 15, 2013). UNDP-HDRO Human Development Reports.

Ni YH, Chang MH, Hsu HY, et al (1991). Hepatocellular carcinoma in childhood. Clinical manifestations and prognosis. Cancer, 68, 1737-41.

Pakzad R, Mohammadian-Hafshejani A, Ghoncheh M, et al (2015a). The incidence and mortality of lung cancer and their relationship to development in Asia. Transl Lung Cancer Res, 4, 763-74.

Pakzad R, Mohammadian-Hafshejani A, Ghoncheh M, et al (2015b). The incidence and mortality of prostate cancer and its relationship with development in Asia. Prostate International, 3, 135-40.

Pakzad R, Mohammadian-Hafshejani A, Mohammadian M, et al $(2015$ c). Incidence and mortality of bladder cancer and their relationship with development in Asia. Asian Pac J

Cancer Prev, 16, 7365-74.

Razi S, Ghoncheh M, Mohammadian-Hafshejani A, et al (2016). The incidence and mortality of ovarian cancer and their relationship with the Human Development Index in Asia. Ecancermedicalscience, 10, 628.

Sagar AD, Najam A (1998). The human development index: a critical review. Ecological Economics, 25, 249-64.

Sepanlou SG, Malekzadeh F, Delavari F, et al (2015). Burden of gastrointestinal and liver diseases in middle east and north africa: results of global burden of diseases study from 1990 to 2010. Middle East J Digestive Dis, 7, 201.

Tobe T, Uchino J, Endo Y, et al (1994). Predictive factors for long term prognosis after partial hepatectomy for patients with hepatocellular carcinoma in Japan. The Liver Cancer Study Group of Japan. Cancer, 74, 2772-80.

Wei K-R, Yu X, Zheng R-S, et al (2014). Incidence and mortality of liver cancer in China, 2010. Chinese J Cancer, 33, 388.

Yamanaka N, Okamoto E, Oriyama T, et al (1994). A prediction scoring system to select the surgical treatment of liver cancer. Further refinement based on 10 years of use. Ann Surg, 219, 342.

Zhang W, Sun B (2015). Impact of age on the survival of patients with liver cancer: an analysis of 27,255 patients in the SEER database. Oncotarget, 6, 633. 